\title{
ON OCCUPATION TIMES FOR MARKOFF PROCESSES
}

BY

\author{
D. A. DARLING AND M. KAC( $\left.{ }^{1}\right)$
}

1. Introduction. Let $x(t), t \geqq 0$, be a Markoff process with stationary transitions and values in an abstract space, and $V(x)$ a non-negative function over that space. In this paper we shall study the limiting distribution of random variables

$$
\frac{1}{u(t)} \int_{0}^{t} V(x(\tau)) d \tau, \quad t \rightarrow \infty,
$$

where $u(t)$ is a suitable normalization. If $V(x)$ is the characteristic function of a set, $\int_{0}^{t} V(x(\tau)) d \tau$ is the occupation time of the set. The principal result is that under suitable (but quite general) conditions the limiting distribution must be the Mittag-Leffler distribution (of an appropriate index).

The method of proof is equally applicable to Markoff chains and, in particular, to sums of independent, identically distributed random variables. We thus obtain a considerable generalization and unification of previous results of Feller [1], Chung and Kac [2] and Kallianpur and Robbins [3;4]. It will be seen that the somewhat lengthy computations of these authors can be dispensed with by virtue of the elementary Tauberian theorem of Karamata.

Finally the distribution of the number of changes of sign in a sequence of partial sums of identically distributed random variables will also emerge as an application of our general theory.

2. A special case. In order to illustrate the method and bring out clearly the role of assumptions under which the general theorem will be proved we shall first consider a special case.

Let $x(t), t \geqq 0$ be the two dimensional Brownian motion, $x(0)=0$, and let $V(x)$ be the characteristic function of a bounded plane set $B$ of nonzero Lebesgue measure. Let us calculate the moments of $\int_{0}^{t} V(x(\tau)) d \tau$. Consider e.g. the second moment

$$
\begin{aligned}
& \mu_{2}(t)=E\left\{\left(\int_{0}^{t} V(x(\tau)) d \tau\right)^{2}\right\}=2 ! \int_{0}^{t} \int_{0}^{\tau_{2}} E\left\{V\left(x\left(\tau_{1}\right)\right) V\left(x\left(\tau_{2}\right)\right)\right\} d \tau_{1} d \tau_{2} \\
& =2 ! \int_{0}^{t} \int_{0}^{\tau_{2}} \int_{-\infty}^{\infty} \int V\left(x_{1}\right) V\left(x_{2}\right) P\left(0 \mid x_{1} ; \tau_{1}\right) P\left(x_{1} \mid x_{2} ; \tau_{2}-\tau_{1}\right) d x_{1} d x_{2} d \tau_{1} d \tau_{2},
\end{aligned}
$$

Received by the editors September 2, 1955 and, in revised form, May 21, 1956.

(1) This research was supported in part by the United States Air Force under contract No. AF 18 (600)-685 monitored by the Office of Scientific Research. 
where

$$
P(x \mid y ; t)=\frac{1}{2 \pi t} e^{-\|x-y\|^{2} / 2 t},
$$

$\|x-y\|$ being the Euclidean distance between $x$ and $y$.

Introducing Laplace transforms we have

$s \int_{0}^{\infty} e^{-8 t} \mu_{2}(t) d t=\frac{2 !}{\pi^{2}} \int_{-\infty}^{\infty} \int V\left(x_{1}\right) V\left(x_{2}\right) K_{0}\left((2 s)^{1 / 2}\left\|x_{1}\right\|\right) K_{0}\left((2 s)^{1 / 2}\left\|x_{2}-x_{1}\right\|\right) d x_{1} d x_{2}$,

where $K_{0}$ is the familiar Bessel function of the second kind appearing in virtue of the well known formula

$$
\int_{0}^{\infty} e^{-8 t} P(x \mid y ; t) d t=\frac{1}{2 \pi} \int_{0}^{\infty} e^{-8 t-\|x-y\|^{2} / 2 t} \frac{d t}{t}=\frac{1}{\pi} K_{0}\left((2 s)^{1 / 2}\|x-y\|\right) .
$$

Using the asymptotic formula

$$
\frac{1}{\pi} K_{0}\left((2 s)^{1 / 2}\|x-y\|\right)=\frac{1}{2 \pi} \log \frac{1}{s}-\frac{1}{\pi} \log \|x-y\|+O(1), \quad s \rightarrow 0,
$$

we obtain immediately

$$
s \int_{0}^{\infty} e^{-s t} \mu_{2}(t) d t \sim \frac{2 !}{(2 \pi)^{2}}\left(\int_{-\infty}^{+\infty} V(x) d x\right)^{2}\left(\log \frac{1}{s}\right)^{2}, \quad s \rightarrow 0,
$$

and by a trivial extension of the above calculations

$$
s \int_{0}^{\infty} e^{-s t} \mu_{k}(t) d t \sim \frac{k !}{(2 \pi)^{k}}\left(\int_{-\infty}^{\infty} V(x) d x\right)^{k}\left(\log \frac{1}{s}\right)^{k}, \quad s \rightarrow 0,
$$

for $k=0,1,2, \cdots$ Here $C=\int_{-\infty}^{\infty} V(x) d x$ denotes, of course, a double integral which in our special case is simply the plane Lebesgue measure of the set $B$. Since $\log 1 / s$ is a slowly varying function we get by Karamata's Tauberian theorem (since $\mu_{k}(t)$ is nondecreasing and $\mu_{k}(0)=0$ )

$$
\mu_{k}(t) \sim \frac{k !}{(2 \pi)^{k}}\left(\int_{-\infty}^{\infty} V(x) d x\right)^{k}(\log t)^{k}, \quad t \rightarrow \infty .
$$

It now follows immediately that

$$
\lim _{t \rightarrow \infty} \operatorname{Prob}\left\{\frac{2 \pi}{C \log t} \int_{0}^{t} V(x(\tau)) d \tau<\alpha\right\}=1-e^{-\alpha}, \quad \alpha \geqq 0 .
$$

A somewhat more inclusive version of this theorem was proved by Kallianpur and Robbins [3] in a different way.

It should be noted that the theorem depends only on the "infinite" part of the asymptotic expansion (2.1) and hence only on relatively superficial 
properties of the process $x(t)$. The deeper properties (closely related to potential theory) are hidden in the "finite" part $-\log \|x-y\| / \pi$.

3. Preliminaries to the general case. The special case considered above suggests a natural set of conditions to be imposed on a general Markoff process with stationary transitions. Let $(\Omega, \mathcal{F})$ be a measurable space and $P(x, E ; t)=\operatorname{Prob}\{x(t+s) \in E \mid x(s)=x\}, x \in \Omega, E \in \mathcal{F}, t>0$, be the transition probability for a Markoff process $x(t), t \geqq 0, x(0)=x_{0}$, with values in $\Omega$. $P(x, E ; t)$ is a measure over $\mathcal{F}$ for fixed $x, t$ and for each $E$ a measurable function in $(x, t)$ (with respect to $\mathcal{F}$ and the Lebesgue sets in $0 \leqq t<\infty$ ).

Then for each $s>0, x$, the Laplace transform

$$
p_{s}(x, E)=\int_{0}^{\infty} e^{-s t} P(x, E ; t) d t
$$

exists and defines a completely additive measure as a function of $E$ over $\mathfrak{F}$. Let $V(x)$ be a measurable, nonnegative function. In the sequel we suppose $P, V$ satisfy the following condition (A):

(A) There exists a function $h(s) \rightarrow \infty, s \rightarrow 0$, and a positive constant $C$ such that

$$
\int \frac{p_{s}(x, d v)}{h(s)} V(y) \rightarrow C, \quad s \rightarrow 0,
$$

the convergence being uniform in $x \in\{\xi \mid V(\xi)>0\}$.

If $V$ can assume negative values and $C=0$ matters are altered radically(f. a recent paper by Dobrušin [6] who considers a one step random Bernoulli walk in one dimension.

In case $\mathrm{V}(x)=\chi_{B}(x)$, the characteristic function of a set $B$, condition (A) requires the existence of $h(s) \rightarrow \infty, s \rightarrow 0,0<Q(B)<\infty$ such that $p_{s}(x, B) / h(s)$ $\rightarrow Q(B)$ uniformly in $x \in B$. This condition is met in the example of $\$ 2$ by the choice $h(s)=(2 \pi)^{-1} \log (1 / s)$, in which case $Q(B)$ is the Lebesgue measure of $B$.

Considering again the second moment $\mu_{2}$ we have, as in the previous example,

$$
\frac{1}{h^{2}(s)} \int e^{-s t} d \mu_{2}(t)=2 ! \int \frac{p_{s}\left(x_{0}, d y\right)}{h(s)} V(y) \int \frac{p_{s}(y, d z)}{h(s)} V(z) \rightarrow 2 ! C^{2}, \quad s \rightarrow 0,
$$

and by an immediate extension for $k=0,1,2, \cdots$,

$$
\int_{0}^{\infty} e^{-s t} d \mu_{k}(t) \sim k^{\prime} C^{k} h^{k}(s), \quad s \rightarrow 0 .
$$

Without further assumptions we cannot apply the Tauberian theorem. However we have immediately the following 
THEOREM 1. If

$$
h(s)=\frac{L(1 / s)}{s^{\alpha}}, \quad 0 \leqq \alpha<1,
$$

where $L(1 / s)$ is slowly varying as $s \rightarrow 0$, then

$$
\begin{aligned}
& \lim _{t \rightarrow \infty} \operatorname{Prob}\left\{\frac{1}{C h(1 / t)} \int_{0}^{t} V(x(\tau)) d \tau<x\right\} \\
& \quad=g_{\alpha}(x)=\frac{1}{\pi \alpha} \int_{0}^{x} \sum_{j=1}^{\infty} \frac{(-1)^{j-1}}{j !} \sin \pi \alpha j \Gamma(\alpha j+1) y^{j-1} d y .
\end{aligned}
$$

In fact from (3.2) and (3.1) it follows by an application of Karamata's Tauberian theorem that

$$
\lim _{t \rightarrow \infty} E\left\{\left(\frac{\int_{0}^{t} V(x(\tau)) d \tau}{C h(1 / t)}\right)^{k}\right\}=\frac{k !}{\Gamma(\alpha k+1)}, \quad k=0,1,2, \ldots
$$

and the numbers $k ! / \Gamma(\alpha k+1)$ are known to be (see Pollard [5]) the moments of the Mittag-Leffler distribution $g_{\alpha}(x)$, which belong to the determinate case. Unless $0 \leqq \alpha \leqq 1$ these numbers are the moments of no distribution. For $\alpha=1$ one gets the degenerate case

$$
\frac{1}{h(1 / t)} \int_{0}^{t} V(x(\tau)) d \tau \rightarrow C, \quad \quad t \rightarrow \infty,
$$

in probability, which is a kind of weak ergodic theorem.

For $\alpha=0$ the Mittag-Leffler distribution (3.3) becomes the exponential distribution $1-e^{-x}, \quad x \geqq 0 ;$ and for $\alpha=1 / 2$ the truncated normal $(\pi)^{-1 / 2} \int_{0}^{x} e^{-y^{2} / 4} d y, x \geqq 0$.

The interesting thing is that Theorem 1 has the following converse:

THEOREM 2. If the process $x(t)$ and the non-negative function $V(x)$ satisfy (A), and if in addition for some normalizing function $u(t)>0$

$$
\lim _{t \rightarrow \infty} \operatorname{Prob}\left\{\frac{1}{u(t)} \int_{0}^{t} V(x(\tau)) d \tau<x\right\}=G(x),
$$

where $G(x)$ is a nondegenerate distribution then

$$
h(s)=L(1 / s) / s^{\alpha}
$$

for some $\alpha, 0 \leqq \alpha<1$, and slowly varying $L(1 / s)$. Hence

$$
G(x)=g_{\alpha}(x / b),
$$


where $b$ is an appropriate constant.

The proof of this theorem will be given in $§ 5$. The following section will be devoted to some auxiliary results of independent interest.

4. Auxiliary results. By a simple change of variable we rewrite the result (3.1) as follows

$$
\lim _{s \rightarrow 0} \int_{0}^{\infty} e^{-t} E\left\{\left(\frac{\int_{0}^{t / s} V(x(\tau)) d \tau}{C h(s)}\right)^{k}\right\} d t=k !
$$

Let now $T$ be a nonnegative random variable such that

$$
\text { Prob }\{T>t\}=e^{-t},
$$

and which is independent of $x(t)$. We can rewrite (4.1) in the form

$$
\lim _{s \rightarrow 0} E\left\{\left(\frac{\int_{0}^{T / s} V(x(\tau)) d \tau}{C h(s)}\right)^{k}\right\}=k !
$$

and hence we have the following

THEOREM 3.

$$
\lim _{s \rightarrow 0} \operatorname{Prob}\left\{\frac{1}{\operatorname{Ch}(s)} \int_{0}^{T / s} V(x(\tau)) d \tau>x\right\}=e^{-x}, \quad x \geqq 0,
$$

or equivalently

$$
\lim _{s \rightarrow 0} \int_{0}^{\infty} e^{-t} \operatorname{Prob}\left\{\frac{1}{C h(s)} \int_{0}^{t / s} V(x(\tau)) d \tau>x\right\} d t=e^{-x}, \quad x \geqq 0 .
$$

As an almost immediate corollary we obtain

THEOREM 4. For every $\lambda>0$

$$
\limsup _{t \rightarrow \infty} \operatorname{Prob}\left\{\frac{1}{C h(\lambda / t)} \int_{0}^{t} V(x(\tau)) d \tau>x\right\} \leqq e^{\lambda} e^{-x},
$$

and

$$
\begin{aligned}
& \underset{t \rightarrow \infty}{\liminf \operatorname{Prob}}\left\{\frac{1}{C h(\lambda / t)} \int_{0}^{t} V(x(\tau)) d \tau>x\right\} \\
& \geqq \frac{e^{-x}-e^{-\lambda}}{1-e^{-\lambda}}
\end{aligned}
$$

We have, using the nonnegativity of $V$, 


$$
\begin{aligned}
\int_{0}^{\infty} e^{-t} & \operatorname{Prob}\left\{\frac{1}{\operatorname{Ch}(s)} \int_{0}^{t / x} V(x(\tau)) d \tau>x\right\} d t \\
& \geqq \int_{\lambda}^{\infty} e^{-t} \operatorname{Prob}\left\{\frac{1}{\operatorname{Ch}(s)} \int_{0}^{t / s} V(x(\tau)) d \tau>x\right\} d t \\
& \geqq \int_{\lambda}^{\infty} e^{-t} \operatorname{Prob}\left\{-\frac{1}{C h(s)} \int_{0}^{\lambda / s} V(x(\tau)) d \tau>x\right\} d t \\
& =e^{-\lambda} \operatorname{Prob}\left\{\frac{1}{\operatorname{Ch}(s)} \int_{0}^{\lambda / s} V(x(\tau)) d \tau>x\right\} .
\end{aligned}
$$

Setting $s=\lambda / t$ and using (4.4) we obtain (4.5). Estimate (4.6) follows in a similar way.

5. Proof of Theorem 2. We are now ready to prove Theorem 2. The proof is carried out in several steps which we separate for the sake of convenience.

$1^{\circ}$. First we show that

$$
0<\liminf _{t \rightarrow \infty} \frac{u(t)}{h(1 / t)} \leqq \limsup _{t \rightarrow \infty} \frac{u(t)}{h(1 / t)}<\infty .
$$

In fact, if

$$
\limsup _{t \rightarrow \infty} \frac{u(t)}{h(1 / t)}=\infty,
$$

we have

$$
\begin{aligned}
\operatorname{Prob}\left\{\frac{1}{u(t)} \int_{0}^{t} V(x(\tau)) d \tau>x\right\} & \\
= & \operatorname{Prob}\left\{\frac{1}{h(1 / t)} \int_{0}^{t} V(x(\tau)) d \tau>x \frac{u(t)}{h(1 / t)}\right\},
\end{aligned}
$$

and by (4.5) $G(x)=0$, contrary to the assumption of nondegeneracy of $G$. Similarly for the positivity of the lim inf.

$2^{\circ}$. Secondly, we show we might as well take

$$
u(t)=\mu_{1}(t)=E\left\{\int_{0}^{t} V(x(\tau)) d \tau\right\} .
$$

From (3.1) and the obvious inequality

$$
s \int_{0}^{\infty} e^{-s t} \mu_{k}(t) d t \geqq s \mu_{k}(\lambda) \int_{\lambda}^{\infty} e^{-s t} d t=e^{-s \lambda} \mu_{k}(\lambda),
$$

we get (setting $\lambda=1 / s$ ) 
or

$$
\limsup _{s \rightarrow 0} \frac{\mu_{k}(1 / s)}{h^{k}(s)} \leqq e k ! C^{k},
$$

$$
\limsup _{t \rightarrow \infty} \frac{\mu_{k}(t)}{h^{k}(1 / t)} \leqq e k ! C^{k} \text {. }
$$

Using (5.1) we have

$$
\limsup _{t \rightarrow \infty} \frac{\mu_{k}(t)}{u^{k}(t)}=\limsup _{t \rightarrow \infty} \frac{\mu_{k}(t)}{h^{k}(1 / t)}\left(\frac{h(1 / t)}{u(t)}\right)^{k}<\infty,
$$

for positive integers $k=1,2, \cdots$.

Since we assumed that the limiting distribution of

$$
\frac{1}{u(t)} \int_{0}^{t} V(x(\tau)) d \tau
$$

exists and since by (5.3) the moments of (5.4) are bounded it follows that the moments approach limits and in particular $(k=1)$

$$
\lim _{t \rightarrow \infty} \frac{\mu_{1}(t)}{u(t)}
$$

exists. Since the limiting distribution is nondegenerate this limit cannot be zero and hence may be taken to be 1 .

$3^{\circ}$. We now start with (4.4) which we rewrite in the equivalent way

$$
\begin{array}{r}
\lim _{s \rightarrow 0} \int_{0}^{\infty} e^{-t} \operatorname{Prob}\left\{\frac{\mu_{1}(t / s)}{C h(s)} \frac{1}{\mu_{1}(t / s)} \int_{0}^{t / s} V(x(\tau)) d \tau<x\right\} d l=1-e^{-x}, \\
x \geqq(.
\end{array}
$$

By an argument used at the beginning of $2^{\circ}$ we get

$$
\limsup _{s \rightarrow 0} \frac{\mu_{1}(t / s)}{C h(s)} \leqq e^{t},
$$

and the functions $\mu_{1}(t / s) / h(s)$ are nondecreasing functions of $t$ for each $s>0$. It follows that a nondecreasing function $f(t)$ and a sequence $s_{n} \rightarrow 0$ can be chosen so that

$$
\lim _{s_{n} \rightarrow 0} \frac{\mu_{1}\left(t / s_{n}\right)}{C h\left(s_{n}\right)}=f(t)
$$

at each continuity point of $f(t)$. It follows also from (5.1) that $f(t)>0$ for $t>0$.

By assumption

$$
\lim _{s_{n} \rightarrow 0} \operatorname{Prob}\left\{\frac{1}{\mu_{1}\left(t / s_{n}\right)} \int_{0}^{t / s_{n}} V(x(\tau)) d \tau<x\right\}=C i(x),
$$


and hence by (5.5)

$$
\int_{0}^{\infty} e^{-\iota} G\left(\frac{x}{f(t)}\right) d t=1-e^{-x}
$$

Let $R$ be a random variable such that

$$
\operatorname{Prob}\{R<t\}=1-e^{-t}
$$

Formula (5.7) can be rewritten in the form

$$
E\left\{G\left(\frac{x}{f(R)}\right)\right\}=1-e^{-x},
$$

or, denoting by $H(y)$ the distribution function of $f(R)$

$$
\int_{0}^{\infty} G(x / y) d H(y)=1-e^{-x} .
$$

By the exponential change of variable $x=e^{x^{\prime}}, y=e^{y^{\prime}}$ we get

$$
\int_{-\infty}^{\infty} G\left(e^{x^{\prime}-y^{\prime}}\right) d H\left(e^{y^{\prime}}\right)=1-e^{-e^{x^{\prime}}}, \quad-\infty<x^{\prime}<\infty .
$$

The left hand side of (5.8) is recognized as the convolution of the distribution functions $G\left(e^{x}\right)$ and $H\left(e^{x}\right)$. Since the characteristic function of the distribution $1-\exp (-\exp x)$ is $\Gamma(1+i \xi) \neq 0$ and since (by assumption) $G$ is given, the distribution function $H$ is uniquely determined. Thus (5.7) determines $f(t)$ uniquely and it follows that (5.6) can be replaced by the stronger statement

$$
\lim _{s \rightarrow 0} \frac{\mu_{1}(t / s)}{C h(s)}=f(t)
$$

$4^{\circ}$. From (5.9) it will now follow simply that $f(t)=t^{\alpha}, 0 \leqq \alpha<1$.

In fact, $f(t)$ being monotonic, is continuous except for at most a denumerable set of points and consequently if $t_{1}, t_{2}$, and $t_{1} t_{2}$ are continuity points of $f$ we have

$$
f\left(t_{1} t_{2}\right)=\lim _{s \rightarrow 0} \frac{\mu_{1}\left(t_{1} t_{2} / s\right)}{C h(s)}=\lim _{s \rightarrow 0} \frac{\mu_{1}\left(t_{1} t_{2} / s\right)}{C h\left(s / t_{2}\right)} \frac{h\left(s / t_{2}\right)}{h(s)} .
$$

Since $f\left(t_{1} t_{2}\right) \neq 0$ and

$$
\lim _{s \rightarrow 0} \frac{\mu_{1}\left(t_{1} t_{2} / s\right)}{C h\left(s / t_{2}\right)}=f\left(t_{1}\right) \neq 0
$$

it follows that 


$$
\lim _{s \rightarrow 0} \frac{h\left(s / t_{2}\right)}{h(s)}=g\left(t_{2}\right)
$$

exists. Thus we obtain

$$
f\left(t_{1} t_{2}\right)=f\left(t_{1}\right) g\left(t_{2}\right),
$$

and it follows trivially that

$$
f(t)=t^{\alpha}
$$

To prove $0 \leqq \alpha<1$ we need only go back to (5.8) and use characteristic functions. Denoting by $\gamma(\xi)$ the characteristic function of $G\left(e^{x}\right)$ we find immediately

$$
\gamma(\xi)=\frac{\Gamma(1+i \xi)}{\Gamma(1+i \alpha \xi)} .
$$

This is the characteristic function of a nondegenerate distribution if and only if $0 \leqq \alpha<1$ in which case the distribution is $g_{\alpha}\left(e^{x}\right)$.

Finally (5.10) implies $h(t)=t^{\alpha} L(t)$, and the proof of Theorem 2 is complete.

6. Corresponding results for Markoff chains and applications to sums of independent random variables. The preceding results can be carried over to Markoff chains. The only difference is that we now use generating functions instead of Laplace transforms. We shall therefore simply state the assumptions and the principal results.

Let $X_{n}, n=0,1,2, \cdots$ be a Markoff chain with transition probabilities

$$
P_{n}(x, E)=\operatorname{Prob}\left\{X_{n+k} \in E \mid X_{k}=x\right\}
$$

and put

$$
p_{z}(x, E)=\sum_{n=0}^{\infty} P_{n+1}(x, E) z^{n}, \quad 0 \leqq z<1 .
$$

Suppose that $V(x)$ is nonnegative, measurable and that, analogous to (A),

$\left(\mathrm{A}^{\prime}\right)$ There exists a function $h(z) \rightarrow \infty, z \rightarrow 1$, and a positive constant $C$ such that

$$
\int \frac{p_{z}(x, d y)}{h(z)} V(y) \rightarrow C,
$$

the convergence being uniform in $x \in\{\xi \mid V(\xi)>0\}$.

Under these assumptions we have the following

TheOREM 5. A necessary and sufficient condition that for some normalizing sequence $u_{n}$ the limiting distribution of 


$$
\frac{1}{u_{n}} \sum_{j=0}^{n} V\left(x_{j}\right)
$$

should exist and be nonsingular is that

$$
h(z)=\frac{1}{(1-z)^{\alpha}} L\left(\frac{1}{1-z}\right)
$$

for some $\alpha, 0 \leqq \alpha<1$, and slowly varying $L$. In case (6.3) is satisfied $u_{n}$ can be taken to be $C h(1-1 / n)$ and the limiting distribution is then the Mittag-Leffler distribution $g_{\alpha}(x)$.

This theorem is simply the discrete analogue of Theorems 1 and 2 .

One of the simplest examples of a Markoff chain with stationary transitions is furnished by the partial sums of identically distributed independent random variables. Let $Y_{1}, Y_{2}, \cdots$ be such variables and set

$$
X_{n}=Y_{1}+Y_{2}+\cdots+Y_{n} .
$$

Let the common distribution of the $Y$ 's be $F(x)$, and $\phi(t)$ their common characteristic function. Denote by $F^{(n)}(x)$ the distribution function of $X_{n}$.

Then we have

$$
\int_{-\infty}^{\infty} p_{z}(x, d y) e^{i t y}=\int_{-\infty}^{\infty} e^{i t y} \sum_{0}^{\infty} z^{n} d F^{(n+1)}(y-x)=\frac{e^{i t x} \phi(t)}{1-z \phi(t)},
$$

and hence if $E$ is a bounded interval $(a, b)$ with $p_{z}(x, a)=p_{z}(x, b)=0$ a standard inversion formula gives

$$
p_{z}(x, E)=\lim _{T \rightarrow \infty} \frac{1}{2 \pi} \int_{-T}^{T} \frac{\psi(t, x, E) \phi(t)}{1-z \phi(t)} d t,
$$

where

$$
\psi(t, x, E)=\int_{E} e^{-i t(y-x)} d y .
$$

Letting $V(x)=\chi_{B}(x)$, the characteristic function of a bounded set $B$, one needs only to determine the behavior of (6.4) when $z \rightarrow 1$ in order to apply Theorem 5 .

It does not seem easy to give the best possible conditions on $\phi$ to insure that $h(z)$ is of the desired form (6.3). However a relatively simple and useful condition can be formulated as follows: If the distribution function of the $Y$ 's has either (a) an absolutely continuous component or (b) has a lattice structure and if, as $t \rightarrow 0$,

$$
\phi(t) \sim 1-|t| r, \quad 1 \leqq \gamma \leqq 2,
$$


then $\left(A^{\prime}\right)$ is satisfied with

$$
\begin{aligned}
h(z)=\frac{1}{\gamma \sin \pi / \gamma} \frac{1}{(1-z)^{1-1 / \gamma}}, & 1<\gamma \leqq 2, \\
h(z)=\frac{1}{\pi} \log \frac{1}{1-z}, & \gamma=1 .
\end{aligned}
$$

Moreover in case (a) one has

$$
C=\text { Lebesgue measure of } B \text {, }
$$

and in case (b)

$$
C=\text { Number of lattice points in } B \text {. }
$$

Let us indicate the proof by considering case (b) and $\gamma=1$, the lattice being of span 1 . Only minor modifications are necessary to treat the other cases. We can assume that $B$ consists of exactly one point $k$, and then setting $E=\{k\}$ we have, analogous to (6.4),

$$
p_{z}(E)=p_{z}(x, E)=\frac{1}{2 \pi} \int_{-\pi}^{\pi} \frac{e^{-i t(k-x)} \phi(t)}{1-z \phi(t)} d t .
$$

It is clear that for each fixed integer $x$, as $z \rightarrow 1$,

$$
p_{z}(E)=\frac{1}{2 \pi} \int_{-\pi}^{\pi} \frac{d t}{1-z \phi(t)}+O(1) .
$$

Now choose $\epsilon>0$ arbitrary and a corresponding $\eta$ such that

$$
|\phi(t)|>1-\epsilon \text { for }|t|<\eta \text {. }
$$

We have

$$
p_{z}(E)=\frac{1}{2 \pi} \int_{-\eta}^{\eta} \frac{d t}{1-z \phi(t)}+O(1)
$$

and by assumption (6.5) we can write $\phi(t)=1-k(t)|t|$ with $k(t) \rightarrow 1, t \rightarrow 0$. In particular $0<m<|k(t)|<M,|t|<\eta$. Set $t=(1-z) \tau$, obtaining

$$
\int_{-\eta}^{\eta} \frac{d t}{1-z \phi(t)}=\int_{-\eta /(1-z)}^{\eta /(1-z)} \frac{d \tau}{1+|\tau| k((1-z) \tau) z} .
$$

Let $\omega(z) \rightarrow 0, z \rightarrow 1$, sufficiently slowly. We can write

$$
\begin{aligned}
& \int_{-\eta /(1-z)}^{\eta /(1-z)} \frac{d \tau}{1+|\tau| k((1-z) \tau) z}=\int_{-\omega(z) /(1-z)}^{\omega(z) /(1-z)} \frac{d \tau}{1+|\tau| k((1-z) \tau) z} \\
& \quad+\int_{-\eta /(1-z)}^{-\omega(z) /(1-z)} \frac{d \tau}{1+|\tau| k((1-z) \tau) z}+\int_{\omega(z) /(1-z)}^{\eta /(1-z)} \frac{d \tau}{1+|\tau| k((1-z) \tau) z} .
\end{aligned}
$$


Since $|k((1-z) \tau)|>m$ the sum of the last two integrals is bounded by

$$
\frac{2}{m} \log \frac{1+m \eta /(1-z)}{1+m \omega(z) /(1-z)} \sim \frac{2}{m} \log \frac{\eta}{\omega(z)} .
$$

The first integral is clearly asymptotic to

$$
2 \log \frac{\omega(z)}{1-z}
$$

and since $\omega(z)$ can be made to go to zero arbitrarily slowly we get

$$
p_{z}(E)=\frac{1}{\pi} \log \frac{1}{1-z}+o\left(\log \frac{1}{1-z}\right) .
$$

7. Applications to the number of changes of sign. Let $Y_{1}, Y_{2}, \cdots$ be independent identically distributed random variables. We assume that

$$
E\left\{\left|Y_{j}\right|\right\}<\infty
$$

and furthermore assume that $Y_{j}$ has a density function $\rho(x)$ whose characteristic function $\phi(t)$ satisfies

$$
\phi(t) \sim 1-|t|^{\gamma}, \quad 1<\gamma \leqq 2, t \rightarrow 0
$$

and is absolutely integrable in $(-\infty, \infty)$. (These assumptions can be relaxed but we choose them for the sake of convenience; actually (7.2) implies (7.1)). Let $S_{n}=Y_{1}+Y_{2}+\cdots+Y_{n}, n=1,2, \cdots$.

If $N_{n}$ is the number of changes of sign in the sequence $S_{1}, S_{2}, \cdots, S_{n}$ it will now appear that the limiting distribution of

$$
\frac{\gamma \sin \pi / \gamma}{E\left\{\left|Y_{j}\right|\right\}} \frac{N_{n}}{n^{1-1 / \gamma}}
$$

is again the Mittag-Leffler distribution of index $1-1 / \gamma$. For $Y$ 's having the symmetric stable distribution of index $\gamma$ this was proved by Chung and Kac [2] but here it will emerge as an application of our general approach.

Consider the vector Markoff chain $\left(S_{0}=0\right)$

$$
W_{0}=\left(S_{0}, S_{1}\right), \quad W_{1}=\left(S_{1}, S_{2}\right), \quad W_{2}=\left(S_{2}, S_{3}\right), \cdots,
$$

and let $E$ be the set in the plane consisting of the second and fourth quadrants: $E=\{x, y \mid x y<0\}$, and $V(x)=\chi_{E}(x)$. Essentially we need only calculate

$$
p_{z}(E)=\sum_{n=0}^{\infty} \operatorname{Prob}\left\{W_{n+1} \in E\right\} z^{n},
$$

which we shall sketch in a formal way, leaving some of the steps to the reader.

We have 


$$
E\left\{e^{i\left(\xi S_{n}+\eta S_{n}+1\right)}\right\}=\phi^{n}(\xi+\eta) \phi(\eta),
$$

and hence

$$
P_{n+1}(E)=\iint_{E} \frac{1}{(2 \pi)^{2}} \iint_{-\infty}^{\infty} e^{-i(\xi x+\eta y)} \phi^{n+1}(\xi+\eta) \phi(\eta) d \xi d \eta d x d y .
$$

Thus

$$
\begin{aligned}
p_{2}(E) & =\iint_{E} \frac{1}{(2 \pi)^{2}} \int_{-\infty}^{\infty} \int \frac{\phi(\xi+\eta) \phi(\eta)}{1-z \phi(\xi+\eta)} e^{-i(\xi x+\eta y)} d \xi d \eta d x d y \\
& =\frac{1}{2 \pi} \iint_{E} \rho(y-x) \frac{1}{2 \pi} \int_{-\infty}^{\infty} \frac{e^{-i u x} \phi(u)}{1-z \phi(u)} d u d x d y .
\end{aligned}
$$

A simple calculation gives

$$
\begin{aligned}
r(u) & =\iint_{E} \rho(y-x) e^{-i u x} d x d y \\
& =\int_{-\infty}^{\infty} \frac{e^{i u x}-1}{i u x}|x| \rho(x) d x .
\end{aligned}
$$

Thus

$$
r(u) \rightarrow \int_{-\infty}^{\infty}|x| \rho(x) d x
$$$$
u \rightarrow 0,
$$

and

$$
p_{z}(E)=\frac{1}{2 \pi} \int_{-\infty}^{\infty} \frac{r(u) \phi(u)}{1-z \phi(u)} d u .
$$

Thus it follows (as before) that $p_{z}(E)$ satisfies $\left(\mathrm{A}^{\prime}\right)$ and

$$
h(z)=\frac{1}{2 \pi} \int_{-\infty}^{\infty} \frac{\phi(u)}{1-z \phi(u)} d u, \quad C=E\left\{\left|Y_{j}\right|\right\} .
$$

The appearance of $E\left\{\left|Y_{j}\right|\right\}$ in the normalizing constant in (7.3) was already noted in [2]. Formula (7.4) provides an explanation of this curious fact.

8. A remark on Karamata's Tauberian theorem. Suppose that $a_{n} \geqq 0$ and as $z \rightarrow 1$,

$$
\sum_{0}^{\infty} a_{n} z^{n} \sim h(z)
$$

where $h(z) \rightarrow \infty, z \rightarrow 1$. Can one infer that 


$$
\sum_{j=0}^{n} a_{j} \sim C h(1-1 / n) ?
$$

If

$$
h(z)=\frac{1}{(1-z)^{\alpha}} L\left(\frac{1}{1-z}\right),
$$

with slowly varying $L$ the answer is affirmative and is given by Karamata's theorem. Our considerations throw some light on the question as to whether (8.3) is also a necessary condition.

Let us restrict ourselves to $h(z)$ of the special form

$$
h(z)=\frac{1}{2 \pi} \int_{-\pi}^{\pi} \frac{\phi(t)}{1-z \phi(t)} d t .
$$

where

$$
\phi(t)=\sum_{-\infty}^{\infty} c_{k} e^{i k t}
$$

with $c_{k} \geqq 0$ and $\sum c_{k}=1$.

Let us now assume that the Tauberian conclusion (8.2) holds not only for $h(z)$ but for all its powers $h^{k}(z), k=1,2, \cdots$. In other words assume that whenever

$$
\sum_{0}^{\infty} a_{n} z^{n} \sim h^{k}(z)
$$$$
z \rightarrow 1
$$

and $a_{n} \geqq 0$, we have

$$
\sum_{0}^{n} a_{j} \sim b_{k} h^{k}(1-1 / n),
$$$$
n \rightarrow \infty \text {. }
$$

It now follows from our reasoning that $h(z)$ must be of the form (8.3). In fact, consider independent random variables $Y_{1}, Y_{2}, \ldots$ such that

$$
\text { Prob }\left\{Y_{j}=k\right\}=c_{k}, \quad k=0, \pm 1, \cdots,
$$

and let the set $E$ consist of the sole integer 0 . If $V(y)$ is the characteristic function of this set we have (analogue of (3.1))

$$
\sum_{0}^{\infty} z^{n} \frac{1}{k !}\left[E\left\{\left(\sum_{0}^{n+1} V\left(S_{j}\right)\right)^{k}\right\}-E\left\{\left(\sum_{0}^{n} V\left(S_{j}\right)\right)^{k}\right\}\right] \sim h^{k}(z)
$$

and hence by (8.4) and (8.5)

$$
E\left\{\left(\sum_{0}^{n} V\left(S_{j}\right) / h(1-1 / n)\right)^{k}\right\} \rightarrow b_{k},
$$$$
n \rightarrow \infty \text {. }
$$ 
Furthermore (by an analogue of the formula which precedes (5.3)) we have $b_{k} \leqq e k !$

It thus follows that the limiting distribution of

$$
\frac{1}{h(1-1 / n)} \sum_{0}^{n} V\left(S_{j}\right)
$$

exists and hence by Theorem $5 h$ must be of the form (8.3).

We have thus demonstrated that, in a certain sense, condition (8.3) is also necessary.

\section{REFERENCES}

1. W. Feller, Fluctuation theory of recurrent events, Trans. Amer. Nath. Soc. vol. 67 (1949) pp. $98-119$.

2. K. L. Chung and M. Kac, Remarks on fluctuations of sums of independent random variables, Memoirs of the American Mathematical Society, No. 6, 1951, 11 pp.

3. G. Kallianpur and H. Robbins, Ergodic property of the Brounian motion process, Proc. Nat. Acad. Sci. U.S.A. vol. 39 (1953) pp. 525-533.

4. - The sequence of sums of independent random variables, Duke Math. J. vol. 21 (1954) pp. 285-307.

5. H. Pollard, The completely monotonic character of the Mittag-Lefler function $E_{a}(-x)$, Bull. Amer. Math. Soc. vol. 54 (1948) pp. 1115-1116.

6. R. L. Dobrusin, Two limit theorems for the simplest random walk on a line, Uspehi Matematixiskih Nauk (N.S.) vol. 10 (1955) pp. 139-146.

UNIVERSITY OF MICHIGAX, ANN ARbor, Mich.

Cornell University, ITHACA, N. Y. 